\title{
THE ROLE OF SENTINEL LYMPH NODE DETECTION IN EARLY GASTRIC CANCER - LITERATURE REVIEW
}

\author{
Nicolae BACALBASA ${ }^{1,2,3 凶}$, Irina BALESCU ${ }^{4}$, Cristian BALALAU ${ }^{2,5}$, Mihaela VILCU $^{2,3}$, \\ Iulian BREZEAN ${ }^{2,3}$ \\ ${ }^{1}$ Center of Excellence in Translational Medicine, Fundeni Clinical Institute, Bucharest, Romania \\ 2 „Carol Davila“ University of Medicine and Pharmacy, Bucharest, Romania \\ 3 "Ion Cantacuzino“ Clinical Hospital, Bucharest, Romania \\ ${ }^{4}$ Ponderas Academic Hospital, Bucharest, Romania \\ 5 „Sf. Pantelimon“ Clinical Hospital, Bucharest, Romania
}

\section{Abstract}

Gastric cancer has been for a long period of time one of the most aggressive malignancies due to the fact that it was usually diagnosed in advanced stages of the disease. However, in the last decades, introduction of upper digestive endoscopy on a wider scale led to a higher detection of this malignancy in earlier stages. Therefore, surgery with curative intent and, in consequence, better long-term outcomes, has been reported. In order to maximize the effect of surgery and to minimize the postoperative complication rates, in the Eastern studies the concept of sentinel lymph node biopsy has been implemented with satisfactory results. This is a literature review of the studies conducted so far on the theme of sentinel lymph node detection in gastric cancer patients.

Keywords: gastric cancer, sentinel lymph node dissection, lymphadenectomy.

\section{Résumé}

Le rôle de la détection du ganglion sentinelle dans le cancer précoce de l'estomac - revue de la littérature

Le cancer gastrique a longtemps été l'un des cancers les plus agressifs, car il était généralement diagnostiqué à un stade avancé de la maladie. Cependant, au cours des dernières décennies, l'introduction de l'endoscopie digestive supérieure à une plus grande échelle a conduit à une détection plus élevée de cette tumeur maligne au cours des stades précoces. Par conséquent, une chirurgie à visée curative et, dans le même temps, de meilleurs résultats à long terme ont été rapportés. Afin de maximiser l'effet de la chirurgie et de minimiser les taux de complications postopératoires, le concept de biopsie du ganglion sentinelle a été mis en œuvre dans les études orientales avec des résultats satisfaisants. Il s'agit d'une revue de la littérature des études menées jusqu'à présent sur le thème de la détection des ganglions sentinelles chez les patients atteints d'un cancer gastrique.

Mots-clés: cancer gastrique, dissection ganglionnaire sentinelle, curage ganglionnaire. 


\section{INTRODUCTION}

In the last decades, it has been widely demonstrated the fact that association of lymph node dissection provides a better outcome of gastric cancer patients; however, the extent of the lymphadenectomy has been a permanent subject of debate. Therefore, while in the Eastern countries, where the national screening programs provide an efficient detection of gastric cancer patients in an early stage of the disease, a less extended lymph node dissection (such as a D1 lymphadenectomy) seems to provide a good control of the disease; in the Western countries, this neoplastic process is unfortunately diagnosed most often in more advanced stages; in consequence, in such cases a more extended lymph node dissection is mandatory in order to control the disease ${ }^{1-3}$. Due to these aspects, a permanent debate between the Eastern and Western countries developed in regard of the extent of lymph node dissection ${ }^{4-6}$.

In this respect, the concept of sentinel lymph node detection has been proposed, initially in the Eastern countries, and later on, but with a lower enthusiasm, in the Western countries. The method, successfully implemented by Cabanas in penile cancer patients and by Morton in malignant melanoma patients, has become nowadays the standard of care in early stage breast cancer as well as in melanoma ${ }^{7-11}$. In breast cancer and malignant melanoma patients, the method presents a particular interest due to the fact that it can provide an efficient staging of the disease and an adequate tailoring of the adjuvant oncologic treatment ${ }^{10-12}$.

\section{Lymphatic spread in gastric cancer}

The development of lymphatic metastases in gastric cancer patients seems to be in close connection with the tumoral stage at the time of diagnosis; therefore, in patients presenting lesions limited to the mucosa, the risk of subsequent lymph node metastases is lower than $10 \%$; in cases presenting submucosal lesions the risk of lymphatic spread reaches $25 \%$, in cases in which the lesion is limited to the muscularis propria the risk of lymph node metastases reaches $40 \%$ while in cases diagnosed with gastric malignancies invading the serosa the risk is augmented to $70 \%{ }^{13,14}$.

Early gastric cancer, defined as a malignant gastric lesion limited to the submucosal layer, has been traditionally treated by radical gastrectomy in association with lymph node dissection; however, the low rates of positive lymph nodes in association with the increased risk of perioperative complications enabled the surgeons to look for a less invasive method in order to treat early gastric cancer patients ${ }^{15,16}$. For example, it is estimated that up to $97 \%$ of cases diagnosed with early gastric cancer will not benefit from extended lymph node dissection, while the perioperative complication rates remain high ${ }^{17}$. In consequence, the concept of sentinel lymph node biopsy was proposed.

In order to find out which are the cases who could benefit most from this method, or, in other words, which are the cases with the lowest risk of lymph node metastases, the Korean team led by Nam et al conducted a study on 119 early gastric cancer patients; the authors demonstrated that the risk of lymph node metastases was significantly associated with the lack of the epithelial mesenchymal transition related markers such as HGF and CD133, as well as with the presence of the diffuse type component and with the presence of lymphatic invasion. Therefore, in such cases, performing a lymph node dissection might be useful while in the other case a less extended procedure seems to be feasible ${ }^{18}$.

\section{Sentinel lymph node biopsy in gastric cancer patients}

In order to provide an efficient detection of the sentinel lymph node in early gastric cancer patients, initially Technetium injection protocol had been proposed; the method consisted of radiocolloid injection in the peritumoral area via an endoscopic approach, one day before surgery.

In order to maximize the results, other authors proposed the blue dye or indocyanine green injection protocol which is performed intraoperatively as a single method or in association with radiocolloid injection, leading to a dual method of detection.

One of the largest studies which demonstrated the efficacy of the dual method in early gastric cancer patients was conducted by Niihara et al and published in $2016^{6}$. The study aimed to investigate the accuracy of the method of sentinel lymph node biopsy in early stage gastric cancer patients, as well as to detect the predictive factors for the false negative results. The Japanese authors introduced in this study 385 patients diagnosed with early stage gastric cancer (stages $\mathrm{T}_{1}-\mathrm{T}_{2 \mathrm{a}} \mathrm{N}_{0} \mathrm{M}_{0}$ ) between 1999 and 2007 and submitted to surgery with curative intent. In all cases the dual method was performed and consisted of Technetium radiocolloid injection one day before surgery in four points around the primary lesion in the submucosal layer, via endoscopic approach followed by intraoperative injection of the blue dye. A positive lymph node was considered whether ten times higher radioactivity when compared to the surrounding tissues was encountered and/or if it presented a blue color (at visual evaluation). After retrieving the identified sentinel lymph node, the procedure continued by 
a conventional D1 or D2 radical gastrectomy, according to the Japanese therapeutic guidelines ${ }^{19}$; sentinel and non-sentinel lymph nodes were analyzed separately and were considered as metastatic if tumoral deposits larger than $0.2 \mathrm{~mm}$ were seen. In cases with lower than $0.2 \mathrm{~mm}$ tumoral deposits, the nodes were considered as presenting isolated tumoral cells. The sentinel nodes were successfully identified in $96.6 \%$ of cases, the rate of detection not being influenced by the type of the surgical approach (classic versus laparoscopic approach). Among the 385 cases, there were 38 patients with lymph node metastases, 34 of them also presenting positive sentinel lymph nodes. In the remaining four cases, a false negative result was demonstrated, all cases being diagnosed with smaller than $4 \mathrm{~cm}$ lesions; however, three of these cases presented muscularis propria invasion. Another interesting observation was the one regarding the location of the sentinel lymph nodes in false positive patients: while in three cases in which the non-sentinel nodes were positive, these nodes were all located within the lymphatic basins of the sentinel node, in one case presenting a large tumor with serosal invasion, the sentinel node was detected at the level of the common hepatic artery while the non-sentinel positive nodes were located along the lesser curvature. Moreover, the rate of sentinel lymph node detection seems to be significantly influenced by the teaching curve, the detection rate being significantly higher in the later period of the study. The stage of the tumor was another important factor affecting the rate of detection, tumors diagnosed in earlier stages being more frequently associated with a successful detection of the sentinel lymph nodes. The multivariate analysis demonstrated that the most important predictive factor for false negative results was represented by the moment when the method was applied (patients submitted to surgery during the learning curve presenting a significantly higher rate of false negative results). As for the long-term results, the authors reported a five-year recurrence free survival rate of $99 \%$ in negative sentinel node patients and of $86.3 \%$ respectively in positive sentinel node patients ${ }^{6}$.

An interesting method which demonstrated the utility of indocyanine green injection in early gastric cancer patients was described by Hironori Ohdaira et al in 2017; the authors proposed preoperative injection of indocyanine green via endoscopy in the four quadrants of the submucosal area, one day before surgery; at the time of surgery the Pinpoint system was used in order to illuminate the region and detect the sentinel lymph nodes after dissecting the gastrocolic ligament. By using this method, the authors reported a successful identification of the sentinel lymph node in six cases; one case presenting a positive lymph node for indocyanine green staining was finally proved to have metastatic deposits ${ }^{20}$.

\section{East versus West again}

While in the Eastern countries the incidence of early gastric cancer reaches $45-51 \%$, in Western countries less than $30 \%$ of cases are diagnosed in this stage. Therefore, some different therapeutic strategies and opinions have been developed. For example, while in Netherlands the first line therapeutic option is represented by radical gastrectomy with extended lymph node dissection, in the Eastern countries this protocol is rarely encountered ${ }^{17,21}$. In consequence, the Eastern scientists have widely investigated and later on introduced in the standard therapeutic protocol the sentinel lymph node biopsy protocol ${ }^{22,23}$.

An interesting review study conducted on the theme of sentinel lymph node biopsy in early gastric cancer patients was led by Lips et al from Maastricht University, The Netherlands ${ }^{17}$. The study included 21 studies and 1314 patients initially diagnosed with stage $T_{1}-T_{3}$ lesions; among these studies, multiple detection methods were used in four papers, while in the other cases only Technetium or detection dye was used. In all cases using Technetium, peritumoral administration in the submucosal layer was performed via endoscopy one day prior surgery, while in cases submitted to local administration of detection dye, the dye was injected in the submucosal or subserous areas at the time of surgery. According to this study, the detection rates of the sentinel lymph nodes ranged between $66.7 \%$ and $100 \%$, the lowest rates being diagnosed in cases submitted to radiocolloid detection with particles size of $500 \mathrm{~nm}$, while the highest rates of detection being achieved in cases submitted to radiocolloid detection with particles ranging between $50 \mathrm{~nm}$ and $100 \mathrm{~nm}$. Another method associated with a higher risk of detection was represented by indocyanine green injection, the detection rates being of $98.8 \%$ : however, no significant association between the tumoral depth and the rates of sentinel lymph node positivity could be established. The authors also revealed the fact that the radiocolloid method was associated with a higher accuracy when compared to dye detection, while the dual method (radiocolloid and dye was associated with the best results). However, the fact that a mean and respectively median sensitivity rate of $85.4 \%$ and $88.9 \%$ respectively was considered not enough to introduce the method as part of the standard therapeutic protocol in the Netherlands; moreover the authors underlined the fact that in other solid organ malignant tumors, the sensitivity of the method had to be higher than $90 \%$ in order to consider it as part of the standard therapeutic protocol. Another aspect 
which was underlined by the Western authors was the one regarding the possible differences in terms of the tumors' characteristics between the Eastern and the Western patients; while in the Eastern patients the tumors are more frequently related to the presence of Helicobacter pylori infection are rather located in the distal third of the stomach, in the Western patients the tumors are rather located at the level of the upper part of the stomach; consequently, the ability of detecting the sentinel lymph nodes seems to be higher in the Eastern patients. The authors concluded that the role of sentinel lymph node biopsy in early gastric cancer patients is still to be established in the Western countries after analyzing the method in large prospective studies conducted in the European countries; in this way, a better tailoring to the type of the Western tumoral subtypes should be provided. In terms of the type of the method, the authors recommended using the dual method (based on both radiocolloid and dye injection); as for the learning curve, the authors concluded that at least 10 cases should be performed in order to achieve a satisfactory sensitivity of the method ${ }^{17}$.

The limits of the method when it comes to the Western patients were also underlined by the Italian authors conducted by Cozzaglio et $\mathrm{al}^{24}$. Similarly to the previous study, the authors pointed out the fact that the method has a low sensitivity rate of sentinel lymph node detection, especially when is performed in the Western patients and underlined the fact that the differences in terms of tumoral subtypes and localization should be taken in consideration when extrapolating the method from the Eastern to the Western patients. The study included 28 cases submitted to sentinel node detection after blue dye injection, the aims of the paper being reporting the rate of successful detection, time until sentinel lymph node detection and number of identified lymph nodes. The authors reported a median time to appearance of 3 minutes and 20 seconds, while the main locations of the nodes were represented by the first stations in 22 cases, the second stations in four cases, one at the first and second levels and one at the first and at the third levels. Sentinel lymph nodes were detected in 28 of the 29 cases, the total number of retrieved nodes being 45 , with a median value of two lymph nodes. Contrarily to the Eastern studies in which the sensitivity rates reached $90 \%$, in the Italian study the sensitivity and specificity of the method were of only $75 \%$, while the positive predictive value was $88 \%$ and the negative predictive value was $55 \%{ }^{24}$. Similarly to the other Western study, the Italian authors pointed out the fact that the more frequent localization of the tumor at the level of the upper pole as well as the higher body mass index of the patients were the important predictive factors for a lower rate of sentinel lymph node detection, when compared to the Eastern studies. Another issue which was discussed by the Italian authors was the relation to the number of the harvested lymph nodes; therefore, while in this study the authors retrieved a reduced number of lymph nodes, considering that the sentinel node is the first node which is identified after injecting the dye, in other Eastern studies all nodes detected at 10-15 minutes after dye injection were considered as sentinel one and retrieved. In consequence, the median number of retrieved nodes was 2 in the Italian study and up to 6 lymph nodes in the Eastern studies ${ }^{25-28}$.

\section{Conclusions}

Sentinel lymph node biopsy seems to be an effective method in order to determine which patients would benefit most after lymph node dissection in early stage gastric cancer, especially in the Eastern countries. Therefore, it seems that the method can be safely and effectively performed in order to minimize the postoperative morbidity rates which might be induced by an extended lymphadenectomy. However, the different locations and characteristics of the gastric lesions led to better results in the Eastern cases when compared to the Western ones.

\section{Compliance with Ethics Requirements:}

„The authors declare no conflict of interest regarding this article"

\section{References}

1. Degiuli M, Sasako M, Ponti A, Calvo F. Survival results of a multicenter phase II study to evaluate D2 gastrectomy for gastric cancer. Br J Cancer. 2004;90(9):1727-1732.

2. Siewert JR, Bottcher K, Roder JD, Busch R, Hermanek P, Meyer HJ. Prognostic relevance of systematic lymph node dissection in gastric carcinoma. German Gastric Carcinoma Study Group. Br J Surg. 1993; 80(8):1015-1018.

3. Bonenkamp JJ, Hermans J, Sasako M, et al. Extended lymph-node dissection for gastric cancer. N Engl J Med. 1999; 340(12):908-914.

4. Maruyama K, Gunven P, Okabayashi K, Sasako M, Kinoshita T. Lymph node metastases of gastric cancer. General pattern in 1931 patients. Ann Surg. 1989; 210(5):596-602.

5. Songun I, Putter H, Kranenbarg EM, Sasako M, van de Velde CJ. Surgical treatment of gastric cancer: 15-year follow-up results of the randomized nationwide Dutch D1D2 trial. Lancet Oncol. 2010;11(5):439-449.

6. Niihara M, Takeuchi H, Nakahara T et al. Sentinel lymph node mapping for 385 gastric cancer patients. J Surg Res. 2016;200(1):73-81. 
7. Cabanas RM. An approach for the treatment of penile carcinoma. Cancer. 1977; 39(2):456-466.

8. Morton DL, Wen DR, Wong JH, et al. Technical details of intraoperative lymphatic mapping for early stage melanoma. Arch Surg. 1992;127(4):392-399.

9. Zahoor S, Haji A, Battoo A, Qurieshi M, Mir W, Shah M. Sentinel lymph node biopsy in breast cancer: a clinical review and update. J Breast Cancer. 2017; 20(3):217-227.

10. Bacalbasa N, Ionescu O, Dimitriu M, Balalau C. Sentinel lymph node biopsy after neoadjuvant chemotherapy in breast cancer with initially cytologically confirmed negative axillary lymph nodes. A review. Arch Balk Med Union. 2017;52(1):62-66.

11. Thomas JM. Sentinel lymph node biopsy in malignant melanoma. BMJ. 2008; 336(7650):902-903.

12. Paraschiv B, Dediu G, Iancu A, Bratu O, Diaconu C. Superior vena cava syndrome - review. Arch Balk Med Union. 2017;52(1):39-43.

13. Akagi T, Shiraishi N, Kitano S. Lymph node metastasis of gastric cancer. Cancers (Basel). 2011;3(2):2141-2159.

14. Folli S, Dente M, Dell'Amore D, et al. Early gastric cancer: prognostic factors in 223 patients. Br J Surg. 1995;82(7):952-956

15. Diaconu C, Balaceanu A, Morosan E. Sepsis biomarkers: past, present and future. Farmacia 2015;63(6):811-815.

16. Bratu OG, Marcu RD, Socea B, et al. Immunohistochemistry particularities of retroperitoneal tumors. Rev Chim (Bucharest). 2018;69(7):1813-1816.

17. Lips DJ, Schutte HW, van der Linden RL, Dassen AE, Voogd AC, Bosscha K. Sentinel lymph node biopsy to direct treatment in gastric cancer. A systematic review of the literature. Eur J Surg Oncol. 2011;37(8):655-661.

18. Nam KH, Yoon H, Lee K, et al. Predictive value for lymph node metastasis of epithelial-mesenchymal transition and cancer stem cell marker expression in early gastric cancer. Pathol Res Pract. 2017;213(9):1221-1226.
19. Japanese Gastric Cancer Association. Japanese classification of gastric carcinoma - 2nd English Edition. Gastric Cancer. 1998;1(1):10-24.

20. Ohdaira H, Yoshida M, Okada S, Tsutsui N, Kitajima M, Suzuki Y. New method of indocyanine green fluorescence sentinel node mapping for early gastric cancer. Ann Med Surg (Lond). 2017; 20:61-65.

21. Socea B, Nica AA, Bratu O, et al. Incidental finding of a sigmoid intussusception associated with rectal prolapse - a case report. Arch Balk Med Union. 2018;53(1):143-146.

22. Pallag A, Rosca E, Tit DM, Mutiu G, Bungau SG, Pop OL. Monitoring the effects of treatment in colon cancer cells using immunohistochemical and histoenzymatic techniques. Romanian Journal of Morphology and Embriology. 2015;56(3):1103-1109.

23. Mogoanta SS, Costache A, Mutiu G, et al. A nonfunctional neuroendocrine tumor of the pancreas - a case report. Romanian Journal of Morphology and Embriology. 2015;56(2 Suppl.):511-519.

24. Cozzaglio L, Bottura R, Di Rocco M, Gennari L, Doci R. Sentinel lymph node biopsy in gastric cancer: possible applications and limits. Eur J Surg Oncol. 2011;37(1):55-59.

25. Kitagawa Y, Fujii H, Mukai M, Kubota T, Otani Y, Kitajima $\mathrm{M}$. Radio-guided sentinel node detection for gastric cancer. Br J Surg. 2002; 89(5):604-608.

26. Miwa K, Kinami S, Taniguchi K, Fushida S, Fujimura T, Nonomura A. Mapping sentinel nodes in patients with early-stage gastric carcinoma. Br J Surg. 2003; 90(2):178-182.

27. Kim MC, Kim HH, Jung GJ, et al. Lymphatic mapping and sentinel node biopsy using $99 \mathrm{mTc}$ tin colloid in gastric cancer. Ann Surg. 2004;239(3):383-387.

28. Uenosono Y, Natsugoe S, Ehi K, Arigami T, Hokita S, Aikou $\mathrm{T}$. Detection of sentinel nodes and micro metastases using radioisotope navigation and immunohistochemistry in patients with gastric cancer. Br J Surg. 2005;92(7):886-889. 